\title{
Future integrated communication network architectures enabling heterogeneous service provision
}

\author{
Paul Arnold and Dirk von Hugo \\ Deutsche Telekom AG, Technology Innovation, Darmstadt 64295, Germany \\ Correspondence: Paul Arnold (paul.arnold@ telekom.de)
}

Received: 25 January 2018 - Accepted: 25 May 2018 - Published: 4 September 2018

\begin{abstract}
This paper summarizes expectations and requirements towards future converged communication systems denoted by 5 th Generation $(5 \mathrm{G})$. Multiple research and standardization activities globally contribute to the definition and specification of an Information and Communication Technology (ICT) to provide business customers and residential users with both, existing and future upcoming services which demand for higher data rates and granted performance figures in terms of QoS parameters, such as low latency and high reliability. Representative use case families are threefold and represented as enhanced Mobile Broadband (eMBB), massive Internet of Things (mIoT), and Critical Communication, i.e. Ultra-Low Latency (ULL)/Ultra-High Reliability (UHR). To deploy and operate a dedicated network for each service or use case separately would raise the expenses and service costs to an unduly high amount. Instead provision of a commonly shared physical infrastructure offering resources for transport, processing, and storage of data to several separated logical networks (slices) individually managed and configured by potentially multiple service providers is the main concept of this new approach.

Beside a multitude of other initiatives the EU-funded $5 \mathrm{G}$ NORMA project (5G Novel Radio Multiservice adaptive network Architecture) has developed an architecture which enables not only network programmability (configurability in software), but also network slicing and Multi Tenancy (allowing independent $3 \mathrm{rd}$ parties to offer an end-to-end service tailored according to their needs) in a mobile network. Major aspects dealt with here are the selectable support of mobility (on-demand) and service-aware QoE/QoS (Quality of Experience/Service) control.

Specifically we will report on the outcome of the analysis of design criteria for Mobility Management schemes and the result of an exemplary application of the modular mobil-
\end{abstract}

ity function to scenarios with variable service requirements (e.g. high-terminal speed vs. on-demand mobility or portability of devices). An efficient sharing of scarce frequency resources in new radio systems demands for tight coordination of orchestration and assignment (scheduling) of resources for the different network slices as per capacity and priority (QoS) demand. Dynamicity aspects in changing algorithms and schemes to manage, configure, and optimize the resources at the radio base stations according to slice specific Service Level Agreements (SLAs) are investigated. It has been shown that architectural issues in terms of hierarchy (centralized vs. distributed) and layering, i.e. separation of control (signaling) and (user) data plane will play an essential role to increase the elasticity of network infrastructures which is in focus of applying SDN (Software Defined Networking) and NFV (Network Function Virtualization) to next generation communication systems.

An outlook towards follow-on standardization and open research questions within different SDOs (Standards Defining Organizations) and recently started cooperative projects concludes the contribution.

\section{Introduction}

In summary the definitions and visions of a future next generation revolutionary new communication system currently denoted by $5 \mathrm{G}$ (5th Generation) is as diverse and dissimilar as all the stakeholders in the ICT (Information and Communication Technology) business are: the heterogeneity in services and technologies reflects the high expectations and requirements towards an ultimate integrated ICT system. Both business customers from areas ranging between automotive industry (autonomous/assisted driving) to medical services 


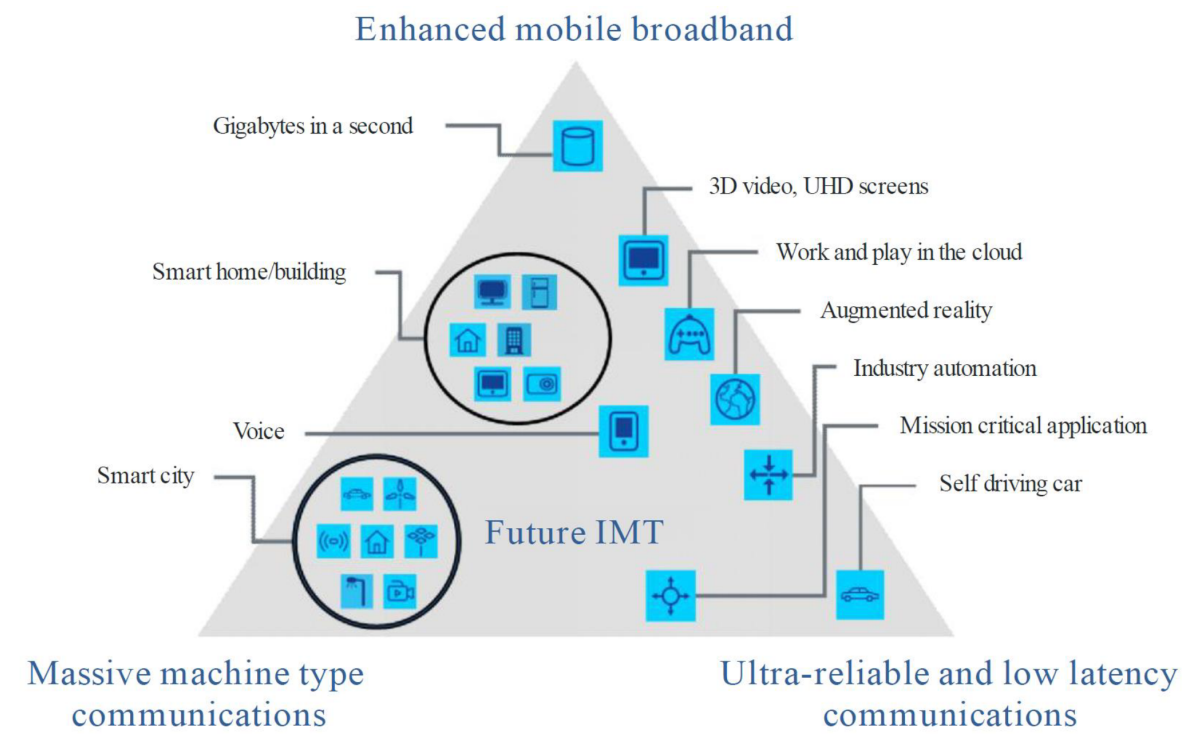

Figure 1. 5G use case families (source: ITU-R, 2015).

(eHealth) and residential users (utilizing speech communication, audio and video streaming, web surfing etc.) will be in focus each to be provided with existing services in a more efficient way but especially also new future upcoming applications with specific demands.

\subsection{G use cases}

Besides the usual increase towards higher data rates (Cisco, 2017) to be supported by new radio (NR) technology and enhancements to LTE-A e.g. in terms of active antenna and interference cancellation techniques the challenge of a $5 \mathrm{G}$ system would be in provision of granted Quality of Service (QoS) such as e.g. very low latency, low error rate, high reliability and reliance. As pointed out in (NGMN, 2015) three use case families have been agreed on as shown in Fig. 1:

- enhanced Mobile Broadband (eMBB): this includes all broadband services as already consumed today via both fixed and wireless access to portable and mobile end terminals such as smart phones and tablets or fixed installed TV screens.

- massive Internet of Things (mIoT) - also denoted as massive Machine Type Communication (mMTC): operation and monitoring of the smart (electricity) grid as well as connected measurement of weather and climate data, but also urban traffic management are typical applications here.

- Critical Communication (CC): this group covers both

- Ultra High Reliability (UHR)

- Ultra Low Latency (ULL) or a combination of both aspects which may be required by industrial applications as remote production machine control or maintenance tasks supported by VR (Virtual Reality).

In general, however, use cases are a mix of multiple features while generally not all requirements have to be met in parallel (e.g. either real-time or high data rate for video, high amount of devices but low data rate for sensor connectivity, etc....)

\subsection{Characteristic new 5G concept: Slicing into virtual sub-networks}

As has been experienced by communication network providers already with preceding deployments of new technologies the essential requirement for every change in a running system is of course a superior performance to meet existing gaps and shortcomings. However, without a business opportunity to monetize, that revenue exceeds the costs in terms of both OPEX and CAPEX (operational/capital expenses) there will be no roll-out. Details on the commercial case for $5 \mathrm{G}$ including potential social benefits and the presentation of a new form of ecosystem can be found in $(5 \mathrm{G}$ NORMA, 2017a).

An approach or rather a concept for the time being, is to provide a service tailored virtualized sub network (slice) for multiple different customers to their specific demands without having to operate a dedicated infrastructure for each service. This is known as logical network slicing: per use case and potentially per vertical tenant (business customer) a logical separated (secured) network is provided which may or may not share resources available in the underlying physical infrastructure. An often used definition of network slic- 


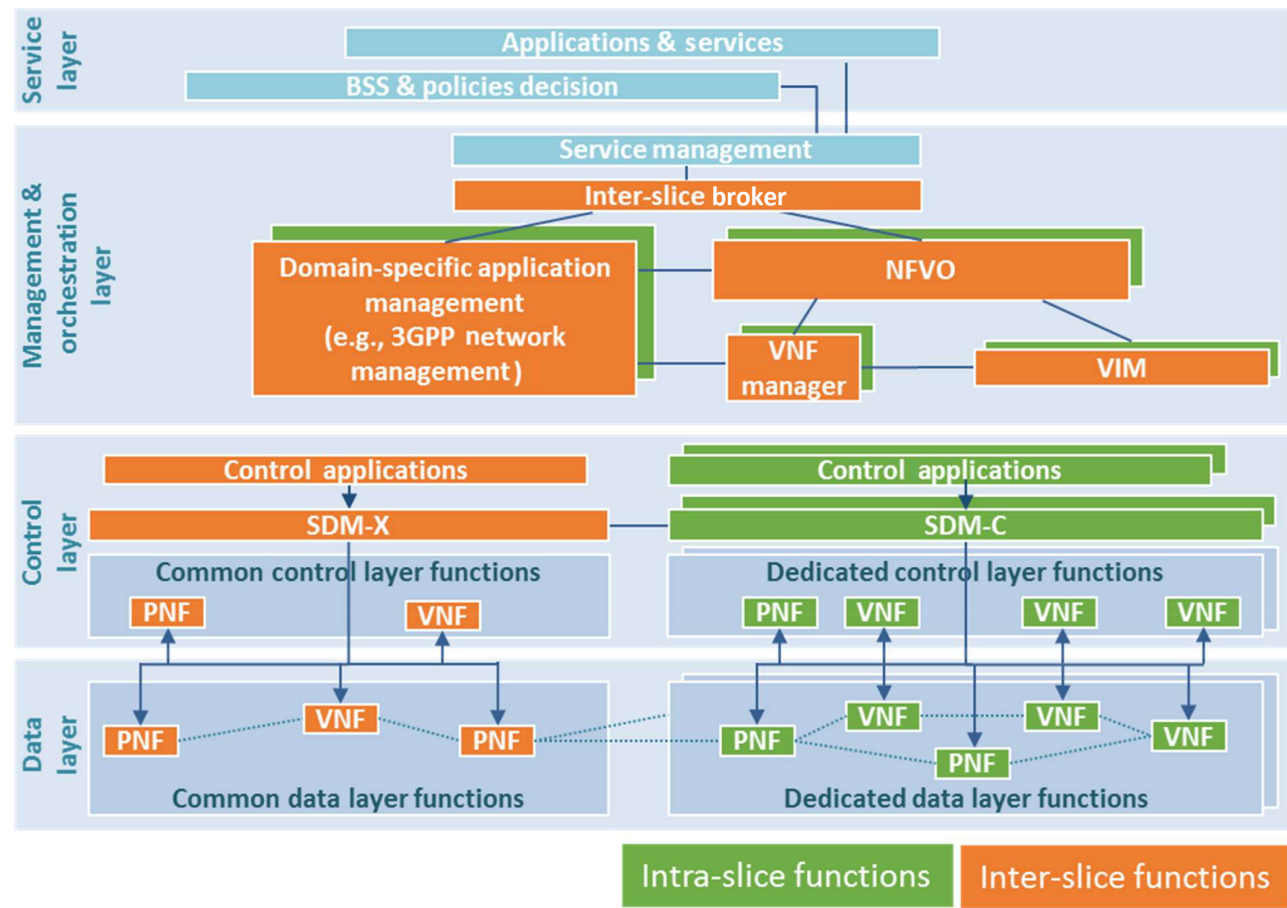

Figure 2. 5G NORMAs Novel Radio Multiservice adaptive network Architecture.

ing is to address network slices as "logically independent networks that run on a common physical infrastructure" (see e.g. GSMA, 2017)

Different slices are distinguished by specifically configured features and service-tailored modular network functions (NFs) in contrast to traditional monolithic network entities (such as MME, S-/P-GW in 3GPP EPC).

\section{Proposed 5G NORMA architecture}

The recently completed EU project 5G NORMA (Novel Radio Multiservice adaptive network Architecture) had as main objective, which consisted of the definition and design of a virtualized, flexible, future-proof, QoE/QoS aware 5G network architecture building on major trends of network "softwarization" and supporting network slicing. The architecture approach followed by 5G NORMA allows for Multi-tenancy and Multi-service support on a single physical infrastructure platform. While multi-tenancy describes the ability to serve multiple service providers from a shared infrastructure set and obtain cost efficiencies via this network sharing approach (i.e. an economies of scale cost benefit), the capability to provide a mix of services with varying quality of service (QoS) requirements is denoted by multi-service support. QoS requirements are detailed e.g. in terms of throughput, reliability, delay, and device density.

\subsection{Software defined Mobile Network (SDM)}

An outline of the proposed network architecture detailed in 5G NORMA is shown in Fig. 2. Such an approach enables for Network Programmability, consists of an SDM Controller (SDM-C) per Logical Slice, of an intelligence dedicated for Management \& Orchestration (MANO), and allows Multi Tenancy support via interfaces to the service layer where decisions on policies made and a BSS (Business Support System) is operated. The (logical) functional entities are realized as VNFs (Virtual Network Functions) following the NFV (Network Function Virtualization) concept as outlined e.g. in (ETSI, 2014). The functions will be deployed as virtual machines or similar hosted within the infrastructure which also consists of (traditional) PNFs (Physical Network Functions) such as antennas, servers etc. For management of resources and configurations of these virtual entities a VIM (Virtual Infrastructure Management) is required which cooperates with the NFV-Orchestrator (NFVO). NFs common for multiple slices sharing the same resources are under control of an SDM-Coordinator (SDM-X).

Key Characteristics of 5G NORMA SDM are beside mentioned multi-tenancy support in terms of capable network management and orchestration

- Dynamic resource sharing between slices which may cover not only radio and transport network resources as described in more detail in Sect. 2.3 but also extend to computation and storage resources within the (virtual) infrastructure shared. Also usage of common data 


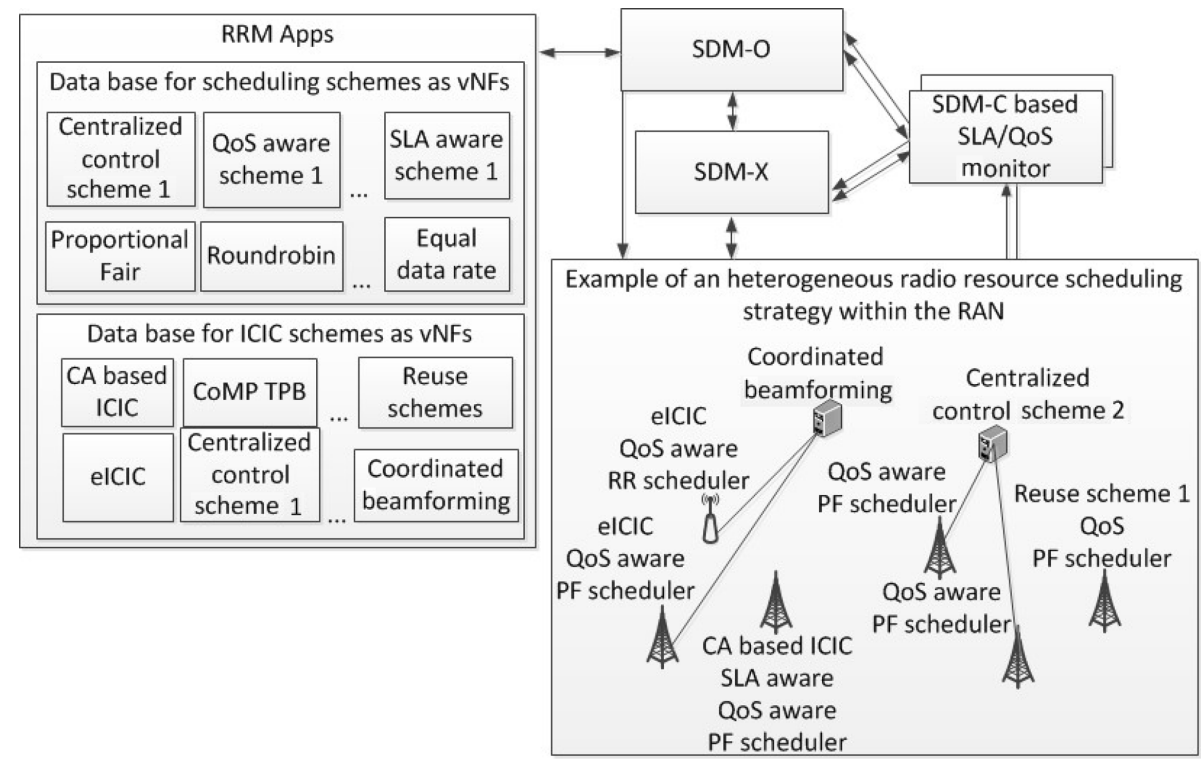

Figure 3. SDM-C/-O/-X interworking for RRM.

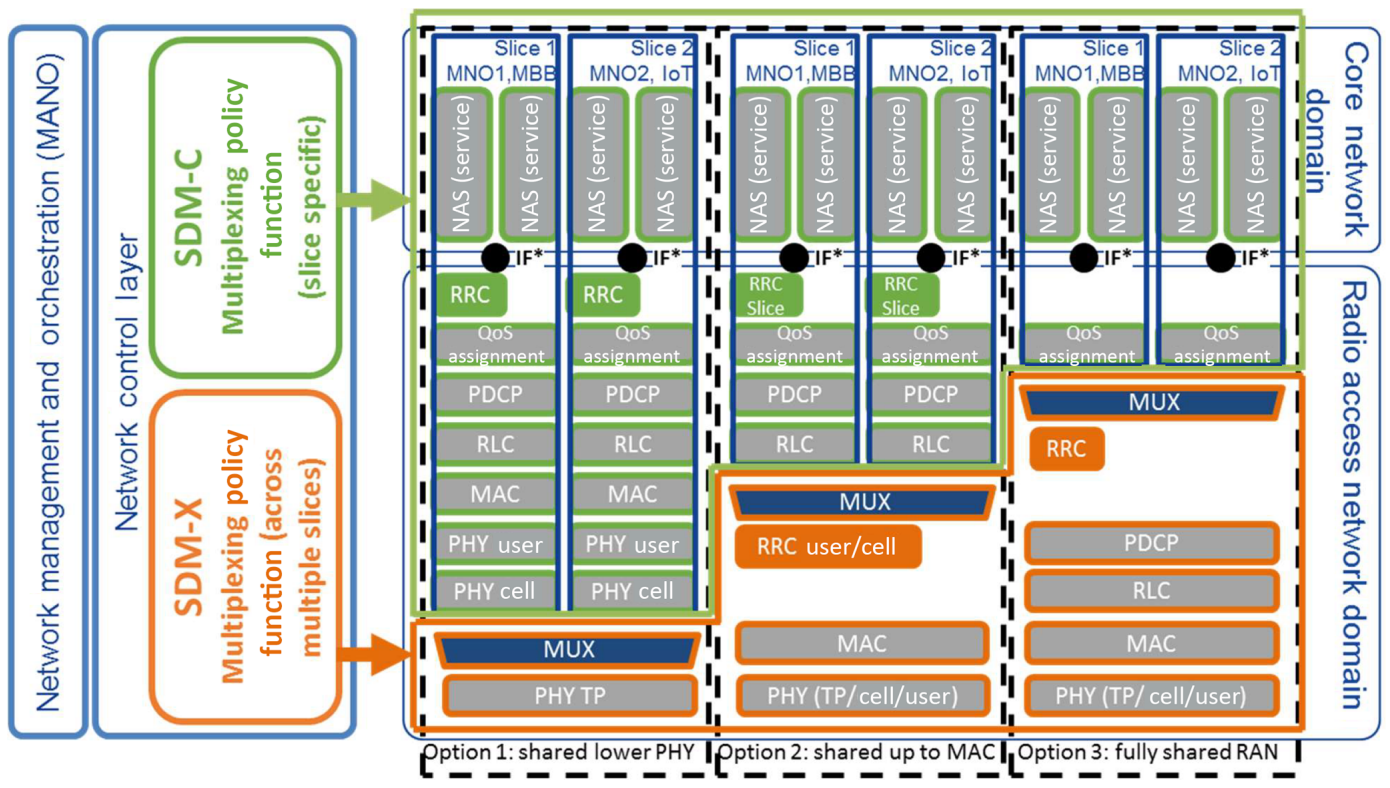

Figure 4. Sharing options on different levels (source: 5G NORMA, 2017a).

files in data centers as for subscription profiles and AAA (Authentication Authorization Accounting) provision to customers using multiple slices' services is considered here (5G NORMA, 2015).

- Separation between common and dedicated network functions will enable to use specifically configured NFs meeting the slices demands as near as possible to avoid any inefficiency which is currently experienced with either multiple identical NFs operated in parallel or over- dimensioned ones providing e.g. high speed mobility support to stationary end devices (5G NORMA, 2015).

- Separation between data layer, control layer, management layer is feature followed by most SDOs since some time such as CUPS (Control and User Plane Separation) in 3GPP 4G core nodes enabling more flexible network deployment and operation. The approach allows for either distributed or centralized deployment and facilitates independent scaling between control plane and user plane functions (e.g. requiring different data rate 


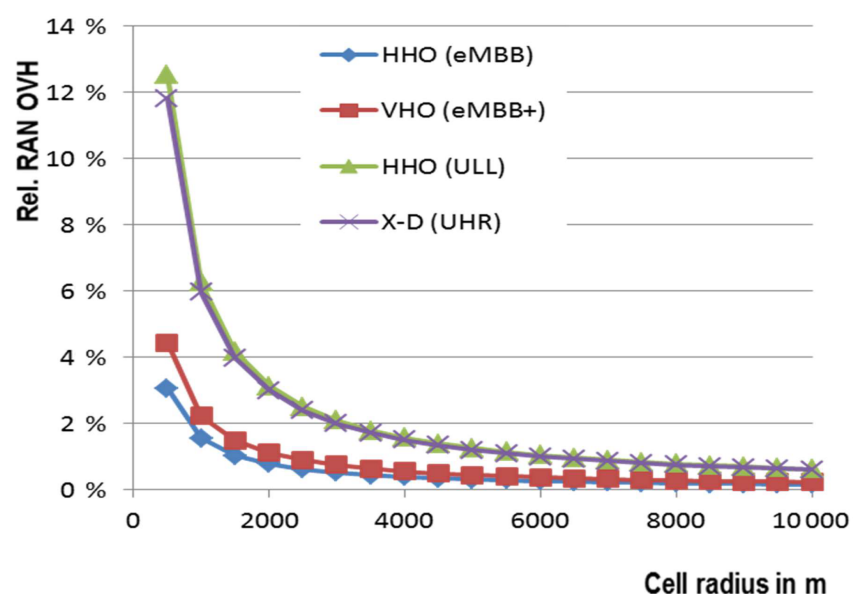

Figure 5. Results for estimated signaling performance per slicespecific mobility demand as a function of cell size.

processing) without impact on proper functioning of existing nodes subject to this split (3GPP, 2017b).

- PNFs and VNFs integrated in common framework and included under SDM-C control is a specific $5 \mathrm{G}$ NORMA challenge, e.g. when equipment at a radio access site is included in mobility operations (e.g. via provision of lower layer information to enhance mobility decisions). In the latter case (since radio frequency in general is assumed to be a shared resource) the PNF would have to be controlled by SDM-X (5G NORMA, 2017d).

\subsection{Functional de-composition: e.g. service-aware mobility and QoS control}

Mobility Management (MM) is one of the features demanding up to now much effort in terms of connectivity and session signaling for a mobile network when compared to a fixed network. Therefore in a future $5 \mathrm{G}$ converged system MM should be available as flexible control application for (on-demand) mobility support of terminals and services only in case it is actually required.

The approach is to design such modular MM for scenarios with variable service requirements ranging from e.g. highspeed movement to nomadic or stationary usage. Other criteria ground on the variety of applications from demanding continuing session preservation to those surviving a session handover or short time outage in case of cellular handoff. Aim is therefore to provide different versions of MM schemes for seam- and lossless session continuity to common schemes with application controlled QoS (i.e. on a higher layer). Details can be found in (von Hugo and Eichler, 2017).

Provision of flexible MM will require a specific sets of VNFs (e.g. anchoring, forwarding, location update ...).

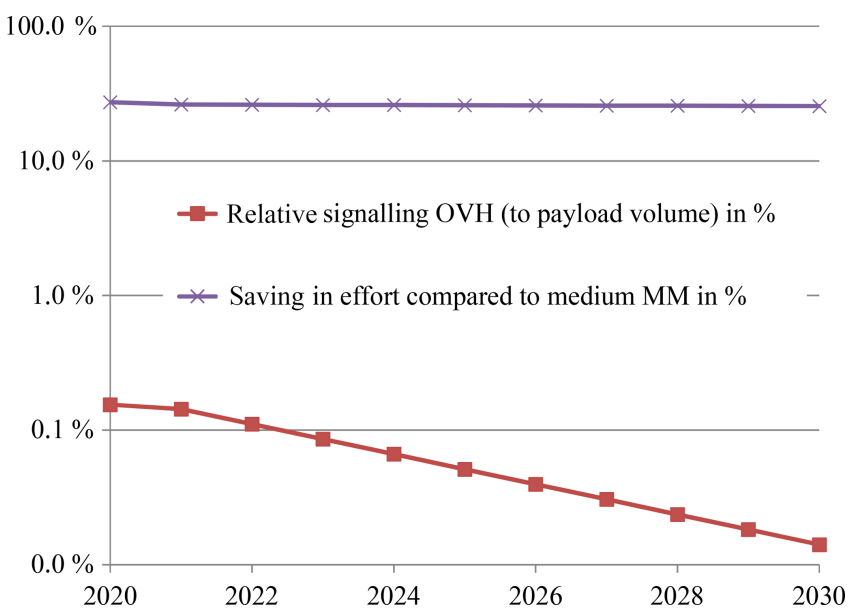

Figure 6. Estimated effort and saving for overall mobility (MM) signaling for a selected scenario as a function of time.

Different approaches have been outlined already in $(5 \mathrm{G}$ NORMA, 2016).

Important to achieve the essential goals of a future $5 \mathrm{G}$ system is to enable efficient sharing of scarce frequency resources in New Radio (NR): possible measures are

- Tight coordinated orchestration and scheduling of resources

- Means to flexibly manage, configure, and optimize resources.

\subsection{Radio aspects - shared resource scheduling with SDM-X}

To meet contradictory SLAs for various tenants, radio resource scheduling strategies might need to be heterogeneously adapted during runtime of network. To introduce a higher flexibility to better control the performance of multiple network slices, a database with virtualized RRM (Radio Resource Management) control applications needs to be defined. The SDM-X, as introduced in Sect. 2.1 needs to react on dynamically appearing critical interference situations in sub areas of the network. Therefore, synchronous inter cell interference coordination (ICIC) schemes need to be influenced by dynamically adapting BS clusters (e.g. for Joint Transmission (JT) or coordinated beamforming). Further the SDM-X can de-/activate asynchronous ICIC schemes (e.g. frequency reuse schemes, carrier aggregation (CA) based ICIC, enhanced (e)ICIC). Additionally it should be possible to de-/active orchestrated alternative Medium Access Control (MAC) scheduling schemes, such as proportional fair, round robin or advanced scheduling metrics. The principle concept is shown in Fig. 3. A subset of the available RRM control applications are instantiated during the orchestration process on the underlying physical infrastructure by the SDM-O. The 


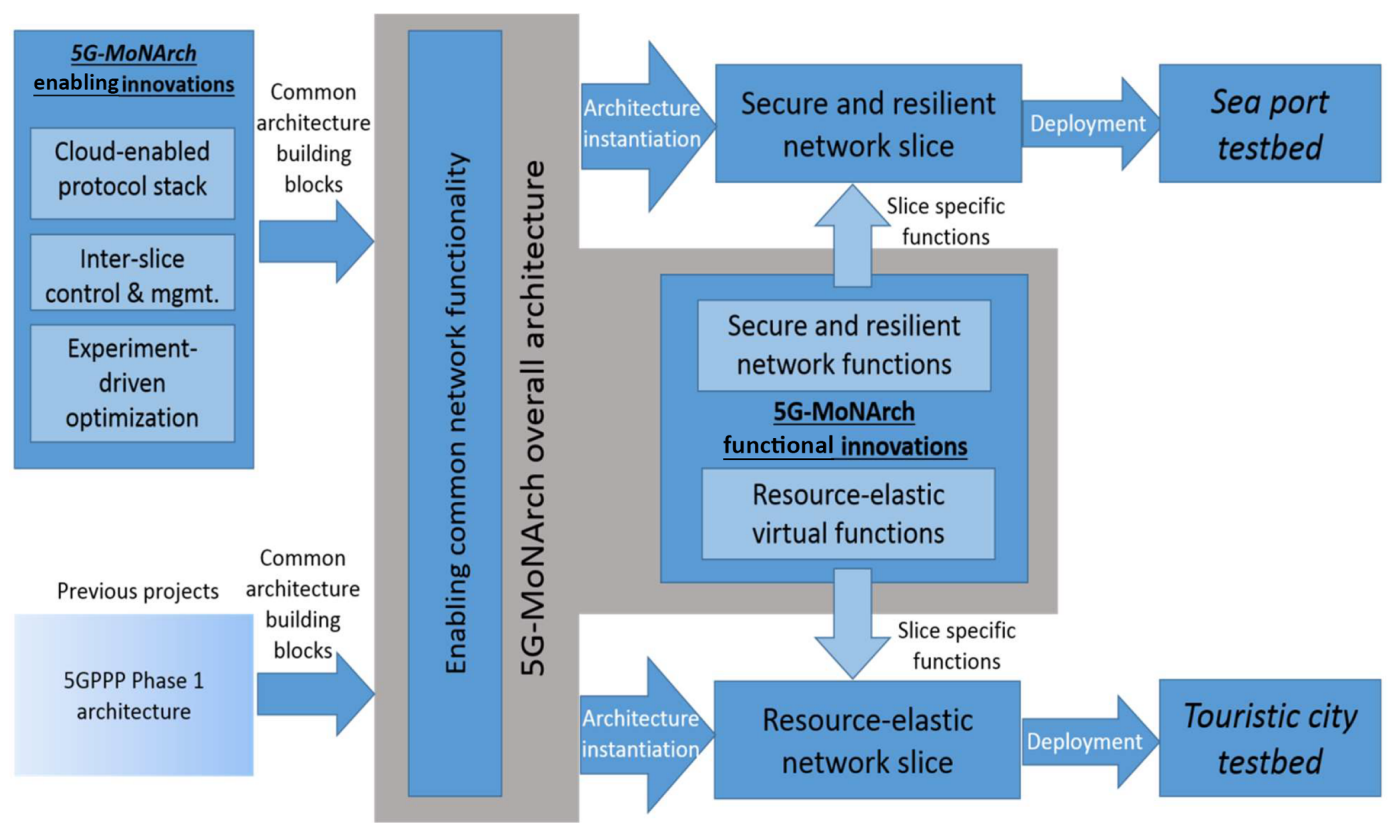

Figure 7. Components and derivation concept of proposed 5G MoNArch architecture.

SDM-X controls the applications during runtime of the network. Further details can be found in (5G NORMA, 2017d).

Another important aspect to support network slicing in the RAN and thus, fulfil contradictory service requirements for different logical networks, is to be able to define slice specific protocol stacks for different slice requirements. This enables three necessary features which allow better adaptation to requirements on the network slice:

- Protocol splits can be implemented on various sublayers of the RAN (3 main options)

- Different levels of slice isolation

- Tenant specific QoS control possible

The mentioned three options to split the radio protocol stack (slice-specific protocol stacks up to including upper PHY, full sharing with dedicated RRC and common MAC) and three solutions for multi-connectivity (common TCP/IP, common PDCP, and common MAC) are discussed as shown in Fig. 4:

- Shared lower PHY

Option 1: RAN slicing with slice-specific RAN stack and shared lower PHY (transmission point specific), with slice specific RRC functionality

- Shared up to MAC

Option 2: RAN slicing with slice-specific PDCP/RLC; with common RRC functionality

- Fully shared RAN
Option 3: RAN slicing with shared RAN (similar to 3GPP Multi-Operator Core Network), with common RRC

Further details can be found in (5G NORMA, 2017c).

\section{Results on mobility scheme adaptation}

A high gain from service-aware mobility scheme selection is expected both for new use cases as mMTC where the devices are in general not moving and for fixed mobile convergence where a wired access network is also attached to the common future $5 \mathrm{G}$ core. Being able to save resource consuming mobility procedures in these cases will cause considerable savings in entity complexity and signaling effort. Details on the approach is presented in (Rates Crippa et al., 2017).

Here we consider that different MM schemes are available which are selected on a-per slice level and thus are supporting different types of handover as e.g.

- Horizontal HandOver/HHO - either in a simple (eMBB) or highly redundant (ULL) manner (i.e. applying multi-path connectivity to at least two points of attachment of the same or different access technologies),

- Vertical HandOver (VHO) between multiple access technologies,

- allowing for cross-Domain (X-D) operation to provide UHR.

Since only specific HO categories need to be considered each MM scheme can be optimized in terms of different relative signaling effort. Parts of the outcome of an exemplary 
quantitative analysis performed in the framework to allow for project achievements' evaluation are depicted in Fig. 5. The shown relation between signaling overhead on the radio interface and the cell size (i.e. frequency of cell change triggered $\mathrm{HO}$ ) is depicted for the four service types. As depicted, savings are achieved for common mobility as simple HHO compared to more complex approaches needed for $\mathrm{X}$-D coordination and the proper choice of the actually required complexity results in an expected enhanced overall efficiency.

For a typical central urban scenario (here: London city) a mix of different services has been assumed for 2020 and beyond (see Fig. 6). As can be detected the overall data volume will increase, but since the type of services assumed will not change the relative signaling overhead will decrease.

As an outcome of the overall evaluation of the architecture and SDMN concepts in project 5G NORMA the introduction of a decoupling between control logic and control agents (enforcement points) in terms of slice-specific controllers and those responsible across multiple slices (SDM$\mathrm{C}$ and SDM-X, respectively) can offer high flexibility to future 5G systems. Being independent from technology- and implementation-related details of network functions for e.g. mobility management, QoE/QoS control, and multi-tenant radio resource management new service-specific dedicated end-to-end networks (slices) can be easily designed and deployed without affecting existing products.

\section{Continuation of the work in follow-up EU project $5 \mathrm{G}$ MoNArch}

Project 5G NORMA was successfully completed end of 2017 but there is still a need for further detailed investigation, such as the required higher efficiency of inter-network slice control and cross-domain management mechanisms. Another issue is the conflict between the design goal of (logically) centralized control functions via SDMC and the requirement for distributed control functions, which results from e.g., latency constraints in the access network. These and similar aspects will be dealt with in a meanwhile started new research project named 5G-MoNArch (5G Mobile Network Architecture) (5G MoNArch, 2017a) where the chosen architecture design is based on principles and concepts proposed by multiple 5G projects including 5G NORMA. The preliminarily architecture of 5G-MoNArch is shown in Fig. 7. Specification of the architecture components is driven by three key enabling innovations shown on the left hand side. The use cases rely on specific functional innovations, such as resource elasticity. Two major testbeds for demonstration and practical experience are planned before 2020 reflecting the main actors in future $5 \mathrm{G}$ service provision, i.e. the vertical industry (use case smart sea port) and the mobile network operator (deployment use case touristic city) (5G MoNArch, 2017b).

\section{Conclusion and next steps}

During the EU funded cooperative research project $5 \mathrm{G}$ NORMA a new and-to-end network concept for $5 \mathrm{G}$ has been proposed and analyzed which allows to support multiple services and tenants according to their specific needs - based on a common jointly used and dynamically configured in frastructure. As was exemplarily shown above the required great flexibility and adaptability to meet demands of future $5 \mathrm{G}$ use cases could be proven as well conceptually as partly by executing computer simulations. The new concept benefits much from the principle of network (i.e. infrastructure resource) sharing between both tenants and providers.

Still some challenges remain to be solved after project completion. These cover especially aspects of planning and operation of the proposed heterogeneous flexible future system. Another ongoing activity is standardization of specifications for global and technological inter-operability at organizations such as e.g. 3GPP. Last but not the least a successful transformation of research results as reported above demands beside a proof of concept in laboratory set-ups also largerscale field trials (testbeds) which can also serve for demonstration. As outlined the fruitful cooperation will continue in other activities such as recently started project 5G MoNArch where the work is further progressed.

Data availability. The underlying research data have been published (5G NORMA, 2017d).

Competing interests. The authors declare that they have no conflict of interest.

Special issue statement. This article is part of the special issue "Kleinheubacher Berichte 2017". It is a result of the Kleinheubacher Tagung 2017, Miltenberg, Germany, 25-27 September 2017.

Acknowledgements. This research work has been performed in the framework of cooperative EU projects. EU funding for H2020-ICT-2014-2 project 5G NORMA (Project ID: 671584) and H2020-ICT-2016-2 project 5G MoNArch (Project ID: 761445) is gratefully acknowledged. The authors would like to acknowledge the support and contributions of their colleagues and partners in the consortia, although the views expressed are those of the authors and do not necessarily represent those of the projects.

Edited by: Jens Anders

Reviewed by: two anonymous referees 


\section{References}

3GPP: 5G Communication for Automation in Vertical Domains, http://www.3gpp.org/news-events/3gpp-news/1839-5g_ cc_automation (last access: 1 June 2018), 2017a.

3GPP: Control and User Plane Separation of EPC nodes (CUPS), http://www.3gpp.org/news-events/3gpp-news/1882-cups (last access: 1 June 2018), 2017b.

5G MoNArch: Project web site, http://cordis.europa.eu/project/rcn/ 211061_en.html and https://5g-monarch.eu/ (last access: 1 June 2018), 2017a.

5G MoNArch: Deliverable D2.1, Baseline architecture based on 5G-PPP Phase 1 results and gap analysis, http://5g-monarch. eu/wp-content/uploads/2017/12/5G-MoNArch_761445_D2. 1_Baseline_Architecture_and_Identified_Gaps_v1.0.pdf (last access: 1 June 2018), 2017b.

5G NORMA: Deliverable D2.1, Use cases, scenarios and requirements, http://www.it.uc3m.es/wnl/5gnorma/pdf/5g_norma_ d2-1.pdf (last access: 1 June 2018), 2015.

5G NORMA: Deliverable D5.1, Definition of connectivity and QoE/QoS management mechanisms - Intermediate report, http:// www.it.uc3m.es/wnl/5gnorma/pdf/5g_norma_d5-1.pdf (last access: 1 June 2018), 2016.

5G NORMA: Deliverable D2.3 Evaluation methodology and KPIs, evaluation architecture design and socio-economic analysis final report, http://www.it.uc3m.es/wnl/5gnorma/pdf/5g_norma_ d2-3.pdf (last access: 1 June 2018), 2017a.

5G NORMA: Deliverable D3.3, 5G NORMA network architecture - Final report, http://www.it.uc3m.es/wnl/5gnorma/pdf/5g norma_d3-3.pdf (last access: 1 June 2018), 2017b.

5G NORMA: Deliverable D4.2, RAN architecture components - Final report, http://www.it.uc3m.es/wnl/5gnorma/pdf/ 5g_norma_d4-2.pdf (last access: 1 June 2018), 2017 c.
5G NORMA: Deliverable D5.2, Definition and specification of connectivity and QoE/QoS management mechanisms - Final report, http://www.it.uc3m.es/wnl/5gnorma/pdf/5g_norma_ d5-2.pdf (last access: 1 June 2018), 2017d.

Cisco VNI: Cisco Visual Networking Index: Global Mobile Data Traffic Forecast Update, 2016-2021 White Paper, http: //www.cisco.com/c/en/us/solutions/collateral/service-provider/ visual-networking-index-vni/mobile-white-paper-c11-520862. html (last access: 1 June 2018), 2017.

GSMA report: Smart 5G networks: enabled by network slicing and tailored to customers' needs, https: //www.gsma.com/futurenetworks/wp-content/uploads/2017/ 09/5G-Network-Slicing-Report.pdf (last access: 1 June 2018), 2017.

ETSI GS NFV 003 V1.2.1: Network Function Virtualisation (NFV); Terminology for Main Concepts in NFV. Group Specification, http://www.etsi.org/deliver/etsi_gs/NFV/001_099/003/ 01.02.01_60/gs_NFV003v010201p.pdf (last access: 1 June 2018), 2014.

ITU-R: IMT Vision -Framework and overall objectives of the future development of IMT for 2020 and beyond, Recommendation ITU-R M.2083-0, https://www.itu.int/dms_pubrec/itu-r/rec/ m/R-REC-M.2083-0-201509-I!!PDF-E.pdf (last access: 1 June 2018), 2015

NGMN: 5G white paper, NGMN Alliance, http://ngmn.org/ 5g-white-paper/5g-white-paper.html (last access: 1 June 2018), 2015.

Rates Crippa, M., Arnold, P., Friderikos, V., Gajic, B., Guerrero, C., Holland, O., Labrador, I., Sciancalepore, V., von Hugo, D., Wong, S., Yousaf, F. Z., and Sayadi, B.: Resource Sharing for a 5G Multi-tenant and Multi-service Architecture, European Wireless, 2017.

von Hugo, D. and Eichler, G.: Distributed Network Infrastructure for Community Services in the Framework of Next Generation Mobile Networks, I4CS 2017, Darmstadt, Germany, 2017. 